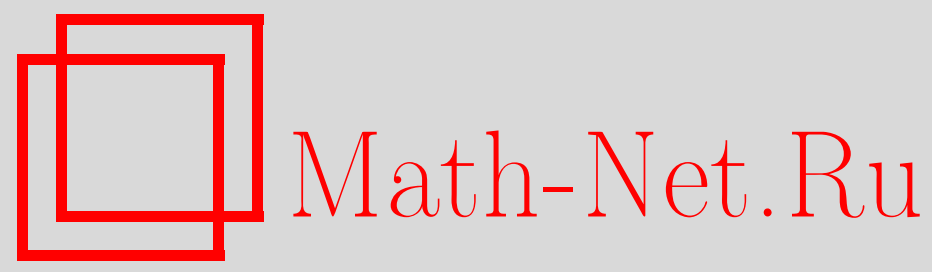

А. С. Соболев, Бесконечномерные гамильтоновы и лагранжевы системы со связями, Матем. заметки, 2002, том 71, выпуск 1, 148-152

DOI: https://doi.org/10.4213/mzm639

Использование Общероссийского математического портала Math-Net.Ru подразумевает, что вы прочитали и согласны с пользовательским соглашением http://www . mathnet.ru/rus/agreement

Параметры загрузки:

IP : 54.224 .60 .19

26 апреля 2023 г., $16: 24: 33$

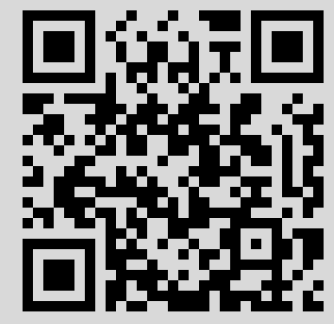




\section{БЕСКОНЕЧНОМЕРНЫЕ ГАМИЛЬТОНОВЫ И ЛАГРАНЖЕВЫ СИСТЕМЫ СО СВЯЗЯМИ}

\section{А. С. Соболев}

В статье предлагается метод, применимый как к лагранжевым, так и к гамильтоновым системам со связями, с помощью которого по лагранжевой системе с (голономными и неголономными) связями может быть получена соответствующая ей система Гамильтона-Дирака (СГД), применение этого же метода к СГД позволяет восстановить лагранжеву систему, эквивалентную исходной. Тем самым, показано, что лагранжевы и гамильтоновы системы со связями являются эквивалентньми способами описания механических систем. Предложенный метод отличается своей универсальностью: в статье он изучается в применении к системам, определенным на рефлексивных банаховых пространствах, однако все используемые формулы имеют смысл и в произвольных локально-выпуклых пространствах. Среди работ по данной тематике можно отметить работы [1]-[5].

1. Основные определения и обозначения. Пусть $X$ и $Y$ - банаховы пространства.

ОПРЕДЕЛЕНИЕ 1. Связи в окрестности точки $x_{0} \in X$ - это отображение $\Phi: X \rightarrow Y$, удовлетворяющее следующим условиям: $\Phi$ один раз непрерывно дифференцируемо по Фреше в некоторой окрестности точки $x_{0}$ и $\Phi\left(x_{0}\right)=0$, отображение $\Phi^{\prime}\left(x_{0}\right): X \rightarrow Y$ сюръективно, множество $\operatorname{Ker} \Phi^{\prime}\left(x_{0}\right)$ дополняемо.

Существуют разложение $X=X_{1} \oplus X_{2}$ и отображение $\varphi: X_{1} \rightarrow X_{2}$ такие, что равенства $\Phi(x)=0$ и $\mathrm{pr}_{X_{2}} x-\varphi\left(\operatorname{pr}_{X_{1}} x\right)=0$ эквивалентны в некоторой окрестности точки $x_{0}$. В этом случае мы будем говорить, что связи $\Phi$ разрешимы относительно подпространства $X_{2}$ с разрешающим отображением $\varphi$. Множество $\Gamma_{\Phi}=\{x \in X: \Phi(x)=0\}$ называется поверхностью связей.

ОПРЕДЕЛЕНИЕ 2. Связи $\Phi_{1}: X \rightarrow Y_{1}$ и $\Phi_{2}: X \rightarrow Y_{2}$ называются эквивалентными в окрестности $O\left(x_{0}\right)$ точки $x_{0} \in \Gamma_{\Phi_{1}}$, если $\Gamma_{\Phi_{1}} \cap O\left(x_{0}\right)=\Gamma_{\Phi_{2}} \cap O\left(x_{0}\right)$.

Пусть $Q$ - реффлексивное банахово пространство, $V=Q$ - его копия, $P=Q^{\prime}$ - пространство линейных непрерьвных функционалов на $Q$. Двойственность между $Q$ и $P$ мы будем обозначать одним из следующих способов: $p(q)=q(p)=p q=(p, q)$.

ОПРЕДЕЛЕНИЕ 3. Лагранжевой системой с неголономными связями на конфигурационном пространстве $Q \times V$ назьвается пара $(L, \Psi)$, где $L: Q \times V \rightarrow \mathbb{R}^{1}-$ дважды непрерьвно дифференцируемая по Фреше функция, назьваемая лагранжианом, $\Psi: Q \times V \rightarrow E_{\Psi}$ - гладкое отображение, удовлетворяющее определению связей и условию неголономности: связи $\Psi$ разрешимы относительно некоторого подпространства пространства $\{0\} \times V$.

ОПРЕДЕЛЕНИЕ 4. Две лагранжевы системы с неголономньми связями $\left(L_{1}, \Psi_{1}\right)$ и $\left(L_{2}, \Psi_{2}\right)$ называются эквивалентными в окрестности $O\left(q^{0}, v^{0}\right)$ точки $\left(q^{0}, v^{0}\right) \in \Gamma_{\Psi_{1}}$, если связи $\Psi_{1}$ и $\Psi_{2}$ эквивалентны в $O\left(q^{0}, v^{0}\right)$ и $L_{1}(q, v)-L_{2}(q, v)=0$ при $(q, v) \in \Gamma_{\Psi_{1}} \cap O\left(q^{0}, v^{0}\right)$.

ОПРЕДЕЛЕНИЕ 5. Гамильтоновой системой со связями (или системой ГамильтонаДирака) на фазовом пространстве $Q \times P$ назьвается пара $(H, \Phi)$, где $H: Q \times P \rightarrow \mathbb{R}^{1}$ - дважды непрерывно дифференцируемая по Фреше функция, называемая гамильтонианом, $\Phi: Q \times P \rightarrow E_{\Phi}-$ гладкое отображение, удовлетворяющее определению связей.

Понятия эквивалентных связей для СГД и эквивалентных СГД определяются аналогично соответствующим понятиям для лагранжевых систем с неголономными связями.

В работе мы будем использовать следующие обозначения: если $P_{1} \subset P$ - дополняемое подпространство, то $p_{1}-$ краткое обозначение для $\mathrm{pr}_{P_{1}} p$; если $P_{1} \oplus P_{2} \subset P$ - дополняемое подпространство, то $P_{1,2}:=P_{1} \oplus P_{2}$ и $p_{1,2}:=\operatorname{pr}_{P_{1} \oplus P_{2}} p=p_{1}+p_{2}$. 
2. Лагранжевы системы с неголономными связями. Далее в статье мы будем рассматривать только лагранжевы системы с неголономными связями, поскольку случай произвольных связей к нему сводится.

Пусть задана лагранжева система с неголономными связями $(L, \Psi)$. В силу неголономности связи $\Psi$ разрешимы относительно некоторого подпространства $\{0\} \times V_{2}$ с разрешающей функцией $\psi$. Дополнение к $V_{2}$ в $V$ обозначим через $V_{0,1}$. Определим связи $\Psi_{0}: Q \times V \rightarrow V_{2}$ равенством $\Psi_{0}(q, v)=v_{2}-\psi\left(q, v_{0,1}\right)$, а лагранжиан $L_{0}: Q \times V_{0,1} \rightarrow \mathbb{R}^{1}$ равенством $L_{0}\left(q, v_{0,1}\right)=L\left(q, v_{0,1}+\right.$ $\left.\psi\left(q, v_{0,1}\right)\right)$. Непосредственно проверяется, что лагранжевы системы $(L, \Psi)$ и $\left(L_{0}, \Psi_{0}\right)$ эквивалентны. Определим функцию $\mathscr{L}: Q \times V \times E_{\Psi^{\prime}}{ }^{\prime} \rightarrow \mathbb{R}^{1}$ равенством $\mathscr{L}(q, v, f)=L(q, v)+\Psi(q, v) f$, $f \in E_{\Psi}{ }^{\prime}$. Будем говорить, что пара функций $(q(\cdot), v(\cdot)), q: \mathbb{R}^{1} \rightarrow Q, v: \mathbb{R}^{1} \rightarrow V$, задает закон изменения состояния механической системы, описываемой данной лагранжевой системой, если найдется функция $f: \mathbb{R}^{1} \rightarrow E_{\Psi}{ }^{\prime}$ такая, что тройка функций $(q(\cdot), v(\cdot), f(\cdot))$ удовлетворяет системе уравнений Эйлера-Лагранжа (СУЭЛ) для функции $\mathscr{L}$, т.е.

$$
\begin{gathered}
\mathscr{L}_{Q}^{\prime}(q(t), v(t), f(t))-\frac{d}{d t} \mathscr{L}_{V}^{\prime}(q(t), v(t), f(t))=0, \\
\Psi(q(t), v(t))=0, \quad v(t)=\frac{d}{d t} q(t) .
\end{gathered}
$$

3. Построение системы Гамильтона-Дирака для лагранжевой системы с неголономными связями. В этом пункте для лагранжевой системы с неголономными связями $(L, \Psi)$ мы построим СГД такую, что между решениями системы уравнений для этой СГД и решениями СУЭЛ для $(L, \Psi)$ можно будет установить взаимно однозначное соответствие. Основой требуемого построения является система

$$
L_{V}^{\prime}(q, v)-p \in \operatorname{Im}\left(\Psi_{V}^{\prime}(q, v)\right)^{*}, \quad \Psi(q, v)=0 .
$$

Лемма 1. Решения системы (1) не изменятся, если лагранжеву систему $(L, \Psi)$ заменить на эквивалентнуло.

Теорема 1. Пусть задана лагранжева система с неголономными связями $(L, \Psi)$. Ecли отображсения $L$ и $\Psi$ удовлетворяют некоторым условиям регулярности, то существуют согласованные разложения пространств $V$ и $P, V=V_{0} \oplus V_{1} \oplus V_{2}, P=P_{0} \oplus P_{1} \oplus P_{2}, u$ решение системы (1) мохно представить в виде $v_{1}=\widehat{v}_{1}\left(q, p_{1,2}, v_{0}\right), v_{2}=\widehat{v}_{2}\left(q, p_{1,2}, v_{0}\right):=$ $\psi\left(q, v_{0}+\widehat{v}_{1}\left(q, p_{1,2}, v_{0}\right)\right), p_{0}=\varphi\left(q, p_{1,2}\right)$.

Мы говорим, что разложение пространства $P=P_{0} \oplus P_{1} \oplus P_{2}$ согласовано с разложением пространства $V=V_{0} \oplus V_{1} \oplus V_{2}$, если выполняются равенства: $P_{0}=\left\{p \in P: \forall v \in V_{1,2} p(v)=0\right\}$, $P_{1}=\left\{p \in P: \forall v \in V_{0,2} \quad p(v)=0\right\}, P_{2}=\left\{p \in P: \forall v \in V_{0,1} p(v)=0\right\}$.

Определим отображение $\widehat{v}: Q \times P_{1,2} \times V_{0} \rightarrow V$ равенством $\widehat{v}\left(q, p_{1,2}, v_{0}\right)=v_{0}+\widehat{v}_{1}\left(q, p_{1,2}, v_{0}\right)+$ $\psi\left(q, v_{0}+\widehat{v}_{1}\left(q, p_{1,2}, v_{0}\right)\right)$. Отображение $\widehat{v}$ удовлетворяет следующим соотношениям, которые устанавливаются при доказательстве теоремы 1 :

$$
\begin{gathered}
L_{V_{0}}^{\prime}\left(q, \widehat{v}\left(q, p_{1,2}, v_{0}\right)\right)=\varphi\left(q, p_{1,2}\right)-\left(\psi_{V_{0}}^{\prime}\left(q, \widehat{v}_{0,1}\left(q, p_{1,2}, v_{0}\right)\right)\right)^{*}\left(L_{V_{2}}^{\prime}\left(q, \widehat{v}\left(q, p_{1,2}, v_{0}\right)\right)-p_{2}\right) \\
L_{V_{1}}^{\prime}\left(q, \widehat{v}\left(q, p_{1,2}, v_{0}\right)\right)=p_{1}-\left(\psi_{V_{1}}^{\prime}\left(q, \widehat{v}_{0,1}\left(q, p_{1,2}, v_{0}\right)\right)\right)^{*}\left(L_{V_{2}}^{\prime}\left(q, \widehat{v}\left(q, p_{1,2}, v_{0}\right)\right)-p_{2}\right) \\
\Psi\left(q, \widehat{v}\left(q, p_{1,2}, v_{0}\right)\right) \equiv 0 .
\end{gathered}
$$

Теперь нетрудно построить СГД для лагранжевой системы $(L, \Psi)$. Определим функцию $\overline{\mathscr{H}}: Q \times$ $P \times V \rightarrow \mathbb{R}^{1}$ равенством $\overline{\mathscr{H}}(q, p, v)=p(v)-L(q, v)$, а функцию $\mathscr{H}: Q \times P \times V_{0} \rightarrow \mathbb{R}^{1}$ равенством $\mathscr{H}\left(q, p, v_{0}\right)=\overline{\mathscr{H}}\left(q, p, \widehat{v}\left(q, p_{1,2}, v_{0}\right)\right)$. Система уравнений Гамилтона-Дирака (СУГД) для функции $\mathscr{H}$, называемой далее обобщенным гамильтонианом, имеет вид

$$
\begin{gathered}
\dot{q}(t)=\mathscr{H}_{P}^{\prime}\left(q(t), p(t), v_{0}(t)\right), \quad \dot{p}(t)=-\mathscr{H}_{Q}^{\prime}\left(q(t), p(t), v_{0}(t)\right), \\
\mathscr{H}_{V_{0}}^{\prime}\left(q(t), p(t), v_{0}(t)\right)=0 .
\end{gathered}
$$

Между решениями СУЭЛ и СУГД существует взаимно однозначное соответствие, которое устанавливается в следующей лемме. В лемме для отображения $L_{0 V_{1}}^{\prime}\left(q, v_{0,1}\right)-\left(\psi_{V_{1}}^{\prime}\left(q, v_{0,1}\right)\right)^{*} p_{2}$ используется краткое обозначение $\widehat{p}_{1}\left(q, v_{0,1}, p_{2}\right)$. Отображение $\widehat{p}_{1}: Q \times V_{0,1} \times P_{2} \rightarrow P_{1}$ является обратным к отображению $\widehat{v}_{1}$, т.е. $\widehat{v}_{1}\left(q, p_{2}+\widehat{p}_{1}\left(q, v_{0,1}, p_{2}\right), v_{0}\right) \equiv v_{1}$. 
Лемма 2. Пусть $(q(\cdot), v(\cdot), f(\cdot))$-решение СУЭЛ. Положим

$$
\begin{aligned}
& p_{2}(\cdot)=\left(\Psi_{V_{2}}^{\prime}(q(\cdot), v(\cdot))\right)^{*} f(\cdot)+L_{V_{2}}^{\prime}(q(\cdot), v(\cdot)) \\
& p_{1}(\cdot)=\widehat{p}_{1}\left(q(\cdot), v_{0,1}(\cdot), p_{2}(\cdot)\right) \\
& p_{0}(\cdot)=\varphi\left(q(\cdot), p_{1}(\cdot)+p_{2}(\cdot)\right)
\end{aligned}
$$

Тогда $\left(q(\cdot), p_{0}(\cdot)+p_{1}(\cdot)+p_{2}(\cdot), v_{0}(\cdot)\right): \mathbb{R}^{1} \rightarrow Q \times P \times V_{0}-$ решение СУГД. Обратно, пусть $\left(q(\cdot), p(\cdot), v_{0}(\cdot)\right)$ - решение СУГД. Положим

$$
\begin{aligned}
& v(\cdot)=\widehat{v}\left(q(\cdot), p_{1,2}(\cdot), v_{0}(\cdot)\right) \\
& f(\cdot)=\left(\left(\Psi_{V_{2}}^{\prime}(q(\cdot), v(\cdot))\right)^{*}\right)^{-1}\left(p_{2}(\cdot)-L_{V_{2}}^{\prime}(q(\cdot), v(\cdot))\right) .
\end{aligned}
$$

Тогда $(q(\cdot), v(\cdot), f(\cdot)): \mathbb{R}^{1} \rightarrow Q \times V \times E_{\Psi}{ }^{\prime}-$ решение СУЭЛ.

Изучим подробнее свойства обобщенного гамильтониана $\mathscr{H}$. Существует отображение $\eta: Q \times$ $V \times P \rightarrow E_{\Psi}{ }^{\prime}$ такое, что $L_{V}^{\prime}\left(q^{0}, p^{0}\right)-p^{0}=\left(\Psi_{V}^{\prime}\left(q^{0}, v^{0}\right)\right)^{*} \eta\left(q^{0}, v^{0}, p^{0}\right)$ для любой точки $\left(q^{0}, v^{0}, p^{0}\right)$, удовлетворяющей системе (1). Функцию $\overline{\mathscr{H}}$ можно представить следующим образом:

$\overline{\mathscr{H}}(q, p, v)=\left(p-L_{V}^{\prime}(q, v)+\left(\Psi_{V}^{\prime}(q, v)\right)^{*} \eta(q, p, v)\right) v+L_{V}^{\prime}(q, v) v-L(q, v)-\left(\Psi_{V}^{\prime}(q, v) v, \eta(q, p, v)\right)$.

Лемма 3. Функция $H_{0}: Q \times P_{1,2} \times V_{0} \rightarrow \mathbb{R}^{1}$, задаваемая равенством

$$
\left.H_{0}\left(q, p_{1,2}, v_{0}\right)=\left.\left(L_{V}^{\prime}(q, v) v-L(q, v)-\left(\Psi_{V}^{\prime}(q, v) v, \eta(q, p, v)\right)\right)\right|_{v=\widehat{v}\left(q, p_{1,2}, v_{0}\right)}\right)
$$

не зависит от $v_{0}$.

Подставляя в $H_{0}$ выражения для $\eta$ и $\widehat{v}$, получим

$$
H_{0}\left(q, p_{1,2}\right)=\left(p_{1,2}, \widehat{v}_{1,2}\left(q, p_{1,2}, v_{0}\right)\right)+\varphi\left(q, p_{1,2}\right) v_{0}-L_{0}\left(q, v_{0}+\widehat{v}_{1}\left(q, p_{1,2}, v_{0}\right)\right)
$$

Из условий, сфформулированных после теоремы 1 , следует справедливость равенства

$$
\left.\left(p-L_{V}^{\prime}(q, v)+\left(\Psi_{V}^{\prime}(q, v)\right)^{*} \eta(q, p, v)\right)\right|_{v=\widehat{v}\left(q, p_{1,2}, v_{0}\right)}=p_{0}-\varphi\left(q, p_{1,2}\right)
$$

Определим отображение $\Phi_{0}: Q \times P \rightarrow P_{0}$ равенством $\Phi_{0}(q, p)=p_{0}-\varphi\left(q, p_{1,2}\right)$. Окончательно получаем, что $\mathscr{H}\left(q, p, v_{0}\right)=H_{0}\left(q, p_{1,2}\right)+\Phi_{0}(q, p) v_{0}$ и лагранжевой системе с неголономными связями $(L, \Psi)$ соответствует СГД $\left(H_{0}, \Phi_{0}\right)$.

4. Восстановление исходной лагранжевой системы по соответствующей ей системы Гамильтона-Дирака. В этом пункте мы применим обратное построение к СГД $(H, \Phi)$, эквивалентной $\left(H_{0}, \Phi_{0}\right)$, и покажем, что резултатом этого построения окажется лагранжева система с неголономными связями $\left(K_{0}, \widetilde{\Psi}\right)$, эквивалентная $(L, \Psi)$. Основой обратного построения является система

$$
H_{P}^{\prime}(q, p)-v \in \operatorname{Im}\left(\Phi_{P}^{\prime}(q, p)\right)^{*}, \quad \Phi(q, p)=0 .
$$

ЛЕмма 4. Для СГД $\left(H_{0}, \Phi_{0}\right)$ и отображения $\widehat{v}$ справедливо равенство

$$
\widehat{v}_{1,2}\left(q, p_{1,2}, v_{0}\right)=H_{0 P_{1,2}}^{\prime}\left(q, p_{1,2}\right)-\left(\varphi_{P_{1,2}}^{\prime}\left(q, p_{1,2}\right)\right)^{*} v_{0}
$$


Tеорема 2. Множества решений систем (1) и (2) совпадают.

Система (2) решается аналогично системе (1); мы построим разложения $V=\widetilde{V}_{0} \oplus \widetilde{V}_{1} \oplus \widetilde{V}_{2}$ и $P=\widetilde{P}_{0} \oplus \widetilde{P}_{1} \oplus \widetilde{P}_{2}$, отличные, вообще говоря, от $V=V_{0} \oplus V_{1} \oplus V_{2}$ и $P=P_{0} \oplus P_{1} \oplus P_{2}$, и найдем решение в виде

$$
\widetilde{p}_{1}=\widehat{\widetilde{p}}_{1}\left(q, \widetilde{v}_{0,1}, \widetilde{p}_{2}\right), \quad \widetilde{p}_{0}=\widehat{\widetilde{p}}_{0}\left(q, \widetilde{v}_{0,1}, \widetilde{p}_{2}\right), \quad \widetilde{v}_{2}=\widetilde{\psi}\left(q, \widetilde{v}_{0,1}\right)
$$

Решения систем (1) и (2) задают одно и то же множество в пространстве $Q \times V \times P$. Равенства $\Psi(q, v)=0$ и $\widetilde{\Psi}(q, v)=0$, где $\widetilde{\Psi}(q, v)=\widetilde{v}_{2}-\widetilde{\psi}\left(q, \widetilde{v}_{0,1}\right)$, задают проекцию этого множества на подпространство $Q \times V \times\{0\}$; следовательно, связи $\Psi$ и $\widetilde{\Psi}$ эквивалентны.

Определим функцию $\overline{\mathscr{K}}(q, v, p)=p(v)-H(q, p)$ и функцию $\mathscr{K}\left(q, v, \widetilde{p}_{2}\right)=\overline{\mathscr{K}}\left(q, v, \widehat{\widetilde{p}}_{0,1}\left(q, \widetilde{v}_{0,1}\right.\right.$, $\left.\left.\widetilde{p}_{2}\right)+\widetilde{p}_{2}\right)$. Повторяя рассуждения из п. 3 , лагранжиан $\mathscr{K}$ можно представить в виде

$$
\mathscr{K}\left(q, v, \widetilde{p}_{2}\right)=\widetilde{p}_{2}\left(\widetilde{v}_{2}-\widetilde{\psi}\left(q, \widetilde{v}_{0,1}\right)\right)+K_{0}\left(q, \widetilde{v}_{0,1}\right),
$$

где $K_{0}$ задается равенством

$$
K_{0}\left(q, \widetilde{v}_{0,1}\right)=v_{2}\left(\widetilde{\psi}\left(q, \widetilde{v}_{0,1}\right)\right)+v_{0,1}\left(\widehat{\widetilde{p}}_{0,1}\left(q, \widetilde{v}_{0,1}, \widetilde{p}_{2}\right)\right)-H\left(q, \widetilde{p}_{2}+\widehat{\widetilde{p}}_{0,1}\left(q, \widetilde{v}_{0,1}, \widetilde{p}_{2}\right)\right)
$$

Лемма 5. Определим $\widehat{\widetilde{p}}\left(q, \widetilde{v}_{0,1}, \widetilde{p}_{2}\right)=\widehat{\widetilde{p}}_{0}\left(q, \widetilde{v}_{0,1}, \widetilde{p}_{2}\right)+\widehat{\widetilde{p}}_{1}\left(q, \widetilde{v}_{0,1}, \widetilde{p}_{2}\right)+\widetilde{p}_{2} \cdot$ Тогдa

$$
\left.\widehat{v}\left(q, p_{1,2}, v_{0}\right)\right|_{p=\widehat{\bar{p}}\left(q, \widetilde{v}_{0,1}, \widetilde{p}_{2}\right), \widetilde{v}_{2}=\widetilde{\psi}\left(q, \widetilde{v}_{0,1}\right)}=\widetilde{v}_{0,1}+\widetilde{\psi}\left(q, \widetilde{v}_{0,1}\right)
$$

Функция $K_{0}$ выражается следующим образом:

$$
K_{0}\left(q, \widetilde{v}_{0,1}\right)=\left.\mathscr{K}\left(q, v, \widetilde{p}_{2}\right)\right|_{\widetilde{v}_{2}=\widetilde{\psi}\left(q, \widetilde{v}_{0,1}\right)} .
$$

Используя это представление и лемму 5 , можно показать, что $K_{0}\left(q, \widetilde{v}_{0,1}\right)=L\left(q, \widetilde{v}_{0,1}+\widetilde{\psi}\left(q, \widetilde{v}_{0,1}\right)\right)$. Итак, из СГД $(H, \Phi)$, эквивалентной СГД $\left(H_{0}, \Phi_{0}\right)$, полученной из лагранжевой системы с неголономньми связями $(L, \Psi)$, нами получена лагранжева система с неголономными связями $\left(K_{0}, \widetilde{\Psi}\right)$, эквивалентная $(L, \Psi)$.

Приложение. Гипотеза Дирака. Решением СУГД мы будем называть пару отображений $(q(\cdot), p(\cdot))$, если найдется отображение $v_{0}: \mathbb{R}^{1} \rightarrow E_{\Phi}{ }^{\prime}$ такое, что тройка $\left(q(\cdot), p(\cdot), v_{0}(\cdot)\right)$ является решением СУГД в обычном смысле. СУГД обладает тем свойством, что если через некоторую точку фазового пространства проходит хотя бы одно решение, то через эту точку проходит, вообще говоря, бесконечное число решений (это верно, если СГД имеет первичные связи первого рода).

ОПРЕДЕЛЕНИЕ 6. Отображение $F: Q \times P \rightarrow E_{F}$ назьвается физической величиной для СГД $(H, \Phi)$, если для любых двух пересекающихся решений $\left(q_{1}(\cdot), p_{1}(\cdot)\right)$ и $\left(q_{2}(\cdot), p_{2}(\cdot)\right)$ СУГД для этой СГД $F\left(q_{1}(t), p_{1}(t)\right) \equiv F\left(q_{2}(t), p_{2}(t)\right)$.

Вообще говоря, не через каждую точку, принадлежащую поверхности связей, проходят решения СУГД (это верно, если СГД имеет вторичные связи), и не для всех отображений $h(\cdot)$ существуют решения СУГД (это верно, если СГД имеет первичные связи второго рода). Задача определения полной поверхности связей (через каждую точку которой проходит решение) и допустимого множества отображений $h(\cdot)$ решается с помошью так назьваемой процедуры определения связей, предложенной Дираком в [3]. В результате этой процедуры определяются новые связи $\Psi$, назьваемые вторичнылми, при этом связи $\Phi$ назьваются первичными. Функцию на фазовом пространстве, скобка Пуассона которой со всеми связями обращается в нуль на полной поверхности связей $\Gamma_{\Phi, \Psi}$, будем называть величиной первого рода. Из связей $\Phi$ и $\Psi$ можно выделить связи первого рода - первичные и вторичные. 
Теорема 3. Пусть $F: Q \times P \rightarrow \mathbb{R}^{1}$ - гладкая функиия. Тогда верно: скобка Пуассона $F$ со всеми связями первого рода обращается в нуль на $\Gamma_{\Phi, \Psi} \Longrightarrow F$ является физической величиной $\Longrightarrow$ скобка Пуассона $F$ с первичными связями первого рода обращается в нуль на $\Gamma_{\Phi, \Psi}$.

Для СГД, принадлежащих классу Дирака, эта теорема допускает усиление, назьваемое гипотезой Дирака. Точное определение класса Дирака слишком громоздко, чтобы приводить его здесь.

ТЕОРема 4. Пусть СГД $(H, \Phi)$ принадлежит классу Дирака, $F: Q \times P \rightarrow \mathbb{R}^{1}-$ гладкая функиия. Тогда $F$ является физической величиной для данной СГД тогда и только тогда, когда скобка Пуассона $F$ со всеми связями первого рода обращается в нуль на $\Gamma_{\Phi, \Psi}$.

\section{СПИСОК ЦИТИРОВАННОЙ ЛИТЕРАТУРЫ}

1. Альбеверио С., Смолянов О. Г. // Докл. РАН. 1997. Т. 355. № 1. С. 7-11. 2. Гитман Д. М., Тютин И.В. Каноническое квантование полей со связями. М.: Наука, 1986. 3. Дирак П.А. М. Принципы квантовой механике. М.: Наука, 1979. 4. Дирак П. А. М. Лекции по теоретической физике. Ижевск: НИЦ Регулярная и хаотическая динамика, 2001. 5. Chernoff P., Marsden J. Properties of Infinite Dimensional Hamiltonian Systems. Lecture Notes in Math. V. 425. New York: Springer-Verlag, 1974.

Московский государственный университет им. М.В. Ломоносова 